TITLE:

\title{
Nonlinear Koopman modes of coupled swing dynamics and coherency identification
}

\author{
$\operatorname{AUTHOR}(\mathrm{S})$ :
}

Susuki, Yoshihiko; Mezi, Igor

\section{CITATION:}

Susuki, Yoshihiko ... [et al]. Nonlinear Koopman modes of coupled swing dynamics and coherency identification. Proceedings of the 2010 IEEE Power \& Energy Society General Meeting (PESGM2010) 2010: 238.

ISSUE DATE:

2010-07

URL:

http://hdl.handle.net/2433/123308

\section{RIGHT:}

(02010 IEEE. Personal use of this material is permitted. However, permission to reprint/republish this material for advertising or promotional purposes or for creating new collective works for resale or redistribution to servers or lists, or to reuse any copyrighted component of this work in other works must be obtained from the IEEE. 


\section{Nonlinear Koopman Modes of Coupled Swing Dynamics and Coherency Identification}

\author{
Yoshihiko Susuki \\ Department of Mechanical Engineering \\ University of California, Santa Barbara \\ CA 93106-5370, United States \\ and \\ Department of Electrical Engineering \\ Kyoto University \\ Nishikyo, Kyoto 615-8510, Japan \\ Email: susuki@ieee.org
}

\author{
Igor Mezić \\ Department of Mechanical Engineering \\ University of California, Santa Barbara \\ CA 93106-5370, United States \\ Email: mezic@engineering.ucsb.edu
}

\begin{abstract}
We report modal analysis of coupled swing dynamics of a multi-machine power system. The analysis is based on the so-called Koopman operator, a linear, infinite-dimensional operator that is defined for any nonlinear dynamical system and captures full information of the system. The modal analysis makes it possible to extract single-frequency modes, which are called Koopman modes, embedded in the coupled swing dynamics and to identify a coherent group of generators in which they swing together in frequency and phase.
\end{abstract}

\section{INTRODUCTION}

Power systems exhibit complex phenomena that occur on a wide range of scales in both space and time. Examples of such phenomena contain synchronization of individual rotating machines, voltage dynamics and collapse, and cascading failures leading to widespread blackouts. Direct numerical simulations of nonlinear mathematical models have demonstrated such complex phenomena, for example, sustained oscillation [1], interarea oscillation [2], chaotic oscillation [3], and cascading failures [4]. Due to high-dimensional, spatiotemporal nature of such phenomena, it is of basic interest for practitioners to identify a small number of dominant components or modes that approximates the phenomena observed practically and numerically. One notion of mode developed in power system analysis is based on small-signal dynamics in which we investigate linearized equations around equilibria. However, since the phenomena listed above do not happen in the neighborhood of equilibria, it is questionable whether global modes for a linearized system are effective for describing such phenomena. Thus, there is a need to develop an alternative approach to mode identification not relying on linearization.

One of the important applications of mode identification is coherency identification in which for transient stability analysis one finds a group of synchronous generators swinging together with the in-phase motion. Objectives of coherency identification include development of low-order models to blackuce computational effort and analysis of instability mechanism (see [5]). Many groups of researchers have developed methods for coherency identification. In [2], [6]-[8], the authors studied the coherency using linear system description and theory. In [9], [10], the authors used the energy function to identify coherent generators. In [11] the authors used the principal component analysis.

In this paper, we develop an alternative method for identification of modes and coherency, by using modal analysis of short-term swing dynamics in a multi-machine power system. Koopman pioneeblack the use of linear transformations on Hilbert space to analyze Hamiltonian systems by introducing the so-called Koopman operator and studying its spectrum [12]: see [13], [14] for details. This linear, infinite-dimensional operator is defined for any nonlinear dynamical systems [13], [14]. Even if the governing dynamics of a system are finitedimensional, the Koopman operator is infinite-dimensional and does not rely on linearization: indeed, it captures the full information of the nonlinear dynamical system. In [15] the authors identified a relationship between general Fourier analysis [16] and eigenfunctions of the Koopman operator. In [17] the author showed via spectral analysis of the Koopman operator that, like in linear dynamics, single-frequency modes can be embedded in highly nonlinear, spatiotemporal dynamics, later named the Koopman Modes (KMs) [18]. In [18] the authors presented a technique for characterizing the global behavior of complex fluid flows by decomposing a flow profile into KMs. In this paper, we apply the technique developed in [17], [18] to shortterm swing dynamics in the New England 39-bus test system (NE system) [19]. We show that the modal analysis makes it possible to extract single-frequency (spatial) modes embedded in coupled swing dynamics of the NE system and to identify a coherent group of generators in which they swing together with the in-phase motion. The identification is performed on finite-time data of the dynamics and does not require the direct check of spatiotemporal patterns. Computation of KM is applicable to dynamics of any power system and can be performed purely on finite-time data of the dynamics. Thus, the identification of modes and coherency based on the KM is suitable for analysis of not only simulation outputs but also data measublack in practice, for example, by wide-area 
measurement [20]. This is the first report of the application of KM to power system analysis. The details of the application are submitted as an archival journal paper [21].

\section{Modal Analysis Based on the KoOpman OPERATOR}

We provides an introduction to the theory of the Koopman operator for nonlinear dynamical systems. The contents in Secs. II-A and II-B are based on [15], [17], [18]. The definitions of the Koopman operator and the Koopman Mode (KM) are presented. an algorithm for computing the KMs from finite data is also presented. Finally we give the definition of coherency in the context of KM.

\section{A. The Koopman Operator, Eigenvalue, and Mode} by

Consider a discrete-time, nonlinear dynamical system given

$$
\boldsymbol{x}_{k+1}=\boldsymbol{F}\left(\boldsymbol{x}_{\boldsymbol{k}}\right),
$$

where $x_{k} \in M$ is the state variable belonging to state space $M$, and $\boldsymbol{F}: M \rightarrow M$ is a nonlinear, vector-valued function. The Koopman operator is a linear operator $\mathcal{U}$ that acts on scalar-valued functions on $M$ in the following manner: for $g: M \rightarrow \mathbb{R}, \mathcal{U}$ maps $g$ into a new function $\mathcal{U} g$ given by

$$
\mathcal{U} g(\boldsymbol{x})=g(\boldsymbol{F}(\boldsymbol{x})) .
$$

Although the dynamical system is nonlinear and evolves on a finite-dimensional space, the Koopman operator $\mathcal{U}$ is linear, but infinite-dimensional. The eigenfunctions and eigenvalues of $\mathcal{U}$ are defined as follows: for functions $\varphi_{j}: M \rightarrow \mathbb{C}$ and constants $\lambda_{j} \in \mathbb{C}$,

$$
\mathcal{U} \varphi_{j}(\boldsymbol{x})=\lambda_{j} \varphi_{j}(\boldsymbol{x}), \quad j=1,2, \ldots
$$

We refer to $\varphi_{j}$ as Koopman eigenfunctions and to $\lambda_{j}$ as the associated Koopman eigenvalues.

The idea in [17] is to analyze dynamics governed by (1) only from available data using the Koopman eigenfunctions and eigenvalues. To this end, consider a vector-valued observable $\boldsymbol{g}: M \rightarrow \mathbb{R}^{p}$. For example, if $\boldsymbol{x} \in M$ contains the full information about system dynamics at a particular time, $\boldsymbol{g}(\boldsymbol{x})$ is a vector of any quantities of interest, such as frequencies and voltages measublack at various points in a power system. If each of the $p$ components of $\boldsymbol{g}$ lies within the span of eigenfunctions $\varphi_{j}$, then as in [17] we may expand $\boldsymbol{g}$ as follows:

$$
\boldsymbol{g}(\boldsymbol{x})=\sum_{j=1}^{K} \varphi_{j}(\boldsymbol{x}) \boldsymbol{v}_{j},
$$

where $K$ is the number of Koopman eigenfunctions and is possibly infinite (in fact, for a finite-dimensional linear system, the number of eigenvalues becomes countably infinite [18]). We will refer to the vectors $\boldsymbol{v}_{j}$ as Koopman Modes (KMs) of the system (1), corresponding to the observable $\boldsymbol{g}$. Then the time series of $\boldsymbol{g}$ on the trajectory of (1) starting at $\boldsymbol{x}_{0}$ at time 0 is given by

$$
\begin{aligned}
\boldsymbol{g}\left(\boldsymbol{x}_{k}\right) & =\sum_{j=1}^{K} \varphi_{j}\left(\boldsymbol{x}_{k}\right) \boldsymbol{v}_{j} \\
& =\sum_{j=1}^{K} \varphi_{j}\left(\boldsymbol{F}\left(\boldsymbol{x}_{k-1}\right)\right) \boldsymbol{v}_{j} \\
& =\sum_{j=1}^{K} \mathcal{U}^{1} \varphi_{j}\left(\boldsymbol{x}_{k-1}\right) \boldsymbol{v}_{j} \\
& =\sum_{j=1}^{K} \mathcal{U}^{2} \varphi_{j}\left(\boldsymbol{x}_{k-2}\right) \boldsymbol{v}_{j} \\
& \vdots \\
& =\sum_{j=1}^{K} \mathcal{U}^{k} \varphi_{j}\left(\boldsymbol{x}_{0}\right) \boldsymbol{v}_{j} \\
& =\sum_{j=1}^{K} \lambda_{j}^{k} \varphi_{j}\left(\boldsymbol{x}_{0}\right) \boldsymbol{v}_{j} .
\end{aligned}
$$

Therefore, the Koopman eigenvalues $\lambda_{j}$ characterize the temporal behavior of the corresponding KM $\boldsymbol{v}_{j}$ : the phase of $\lambda_{j}$ determines its frequency, and the magnitude determines the growth rate. Note that in [17] for a system evolving on an attractor, the Koopman eigenvalues always lie on the unit circle.

We note from [17] that the terms $\varphi_{j}\left(\boldsymbol{x}_{0}\right) \boldsymbol{v}_{j}$ in KMs are associated with a projection operation associated with $\mathcal{U}$ applied to the observable $\boldsymbol{g}$. Define a family of operators $\mathcal{P}^{\nu}$ : for $g: \mathbb{R}^{n} \rightarrow \mathbb{R}$

$$
\mathcal{P}^{\nu} g\left(\boldsymbol{x}_{0}\right)=\lim _{n \rightarrow \infty} \frac{1}{n} \sum_{k=0}^{n-1} \mathrm{e}^{\mathrm{i} 2 \pi k \nu} g\left(\boldsymbol{x}_{k}\right),
$$

where $\nu \in[-1 / 2,1 / 2)$. When the trajectory starting at $\boldsymbol{x}_{0}$ is on an attractor of (1), a nonzero $\mathcal{P}^{\nu}$ is the orthogonal projection operator onto the eigenspace of $\mathcal{U}$ associated with the Koopman eigenvalue $\lambda=\mathrm{e}^{-\mathrm{i} 2 \pi \nu}$. The projections of the $p$ components $g_{1}, \ldots, g_{p}$ of $\boldsymbol{g}$ on the $j$-th eigenspace are obtained:

$$
\left[\begin{array}{c}
\mathcal{P}^{\nu_{j}} g_{1}\left(\boldsymbol{x}_{0}\right) \\
\vdots \\
\mathcal{P}^{\nu_{j}} g_{p}\left(\boldsymbol{x}_{0}\right)
\end{array}\right]=\varphi_{j}\left(\boldsymbol{x}_{0}\right) \boldsymbol{v}_{j}
$$

where $\nu_{j}=\operatorname{Im}\left[\ln \lambda_{j}\right] / 2 \pi$. This formula (3) associates $\varphi_{j}\left(\boldsymbol{x}_{0}\right) \boldsymbol{v}_{j}$ with the projection operation based on the operator $\mathcal{P}^{\nu}$. Apparently the left-hand sides of (3) are just the Fourier transforms of the time series, and the terms $\varphi_{j}\left(\boldsymbol{x}_{0}\right) \boldsymbol{v}_{j}$ can be easily computed.

Also note from [17] that, when the trajectory starting at $\boldsymbol{x}_{0}$ is on an attractor of (1), the general expression of $\boldsymbol{g}\left(\boldsymbol{x}_{k}\right)$ is given as follows:

$$
\boldsymbol{g}\left(\boldsymbol{x}_{k}\right)=\sum_{j=1}^{K} \lambda_{j}^{k} \varphi_{j}\left(\boldsymbol{x}_{0}\right) \boldsymbol{v}_{j}+\int_{\mathbb{T}^{1}} \mathrm{e}^{\mathrm{i} 2 \pi \theta} \mathrm{d} E(\theta) \boldsymbol{g}\left(\boldsymbol{x}_{0}\right),
$$


where $E(\theta)$ is a continuous, complex spectral measure on $L^{2}$. The last term on the right-hand side represents the contribution of continuous spectrum of $\mathcal{U}$ to the observations $\left\{\boldsymbol{g}\left(\boldsymbol{x}_{k}\right)\right\}$ for $k \in \mathbb{N}$.

\section{B. Computation of the Koopman Eigenvalues and Modes}

We numerically compute the Koopman eigenvalues and modes using the Arnoldi algorithm. Suppose that we have a sequence of $N+1$ observations $\left\{\boldsymbol{g}\left(\boldsymbol{x}_{0}\right), \ldots, \boldsymbol{g}\left(\boldsymbol{x}_{N}\right)\right\}$. Let us define the empirical Ritz values $\tilde{\lambda}_{j}$ and empirical Ritz vectors $\tilde{\boldsymbol{v}}_{j}$ of this sequence by using the following algorithm:

(i) Define constants $c_{j}$ such that for vector $r$ satisfying $\boldsymbol{r} \perp \operatorname{span}\left\{\boldsymbol{g}\left(\boldsymbol{x}_{0}\right), \ldots, \boldsymbol{g}\left(\boldsymbol{x}_{N-1}\right)\right\}$,

$$
\boldsymbol{r}=\boldsymbol{g}\left(\boldsymbol{x}_{N}\right)-\sum_{j=0}^{N-1} c_{j} \boldsymbol{g}\left(\boldsymbol{x}_{j}\right) .
$$

(ii) Define the companion matrix $\mathrm{C}$ as

$$
\mathbf{C}=\left[\begin{array}{ccccc}
0 & 0 & \cdots & 0 & c_{0} \\
1 & 0 & \cdots & 0 & c_{1} \\
0 & 1 & \cdots & 0 & c_{2} \\
\vdots & \vdots & \ddots & \vdots & \vdots \\
0 & 0 & \cdots & 1 & c_{N-1}
\end{array}\right]
$$

and find its $N$ eigenvalues $\tilde{\lambda}_{1}, \ldots, \tilde{\lambda}_{N}$.

(iii) Define the Vandermonde matrix $\mathrm{T}$ using $\tilde{\lambda}_{j}$ as

$$
\mathrm{T}=\left[\begin{array}{ccccc}
1 & \tilde{\lambda}_{1} & \tilde{\lambda}_{1}^{2} & \ldots & \tilde{\lambda}_{1}^{N-1} \\
1 & \tilde{\lambda}_{2} & \tilde{\lambda}_{2}^{2} & \cdots & \tilde{\lambda}_{2}^{N-1} \\
\vdots & \vdots & \vdots & \ddots & \vdots \\
1 & \tilde{\lambda}_{N} & \tilde{\lambda}_{N}^{2} & \cdots & \tilde{\lambda}_{N}^{N-1}
\end{array}\right]
$$

(iv) Define $\tilde{\boldsymbol{v}}_{j}$ to be the columns of $\mathrm{V}=$ $\left[\boldsymbol{g}\left(\boldsymbol{x}_{0}\right) \boldsymbol{g}\left(\boldsymbol{x}_{1}\right) \cdots \boldsymbol{g}\left(\boldsymbol{x}_{N-1}\right)\right] \mathrm{T}^{-1}$.

Then, we have the following equations that are originally derived in [18]:

$$
\left.\begin{array}{l}
\boldsymbol{g}\left(\boldsymbol{x}_{k}\right)=\sum_{j=1}^{N} \tilde{\lambda}_{j}^{k} \tilde{\boldsymbol{v}}_{j}, \\
\boldsymbol{g}\left(\boldsymbol{x}_{N}\right)=\sum_{j=1}^{N} \tilde{\lambda}_{j}^{N} \tilde{\boldsymbol{v}}_{j}+\boldsymbol{r},
\end{array}\right\}
$$

where $k=0 \ldots, N-1$. Comparing with (2), the empirical Ritz values $\tilde{\lambda}_{j}$ and vectors $\tilde{\boldsymbol{v}}_{j}$ precisely behave in the same manner as the Koopman eigenvalues and modes, but for the finite sum (5) instead of the (possibly) infinite sum (2).

\section{Coherency in the Koopman Mode}

Finally we define the notion of coherency in the context of KM. The case of oscillatory KM, in which the Koopman eigenvalue has an imaginary part, is addressed, because the study on coherency identification in power systems normally deals with oscillatory responses following a disturbance. For an oscillatory $\mathrm{KM} \boldsymbol{v}_{j}$, called Mode $j$, with the Koopman eigenvalue $\lambda_{j}=r_{j} \mathrm{e}^{\mathrm{i} 2 \pi \nu_{j}}$ and its complex conjugate $\lambda_{j}^{\mathrm{c}}=$

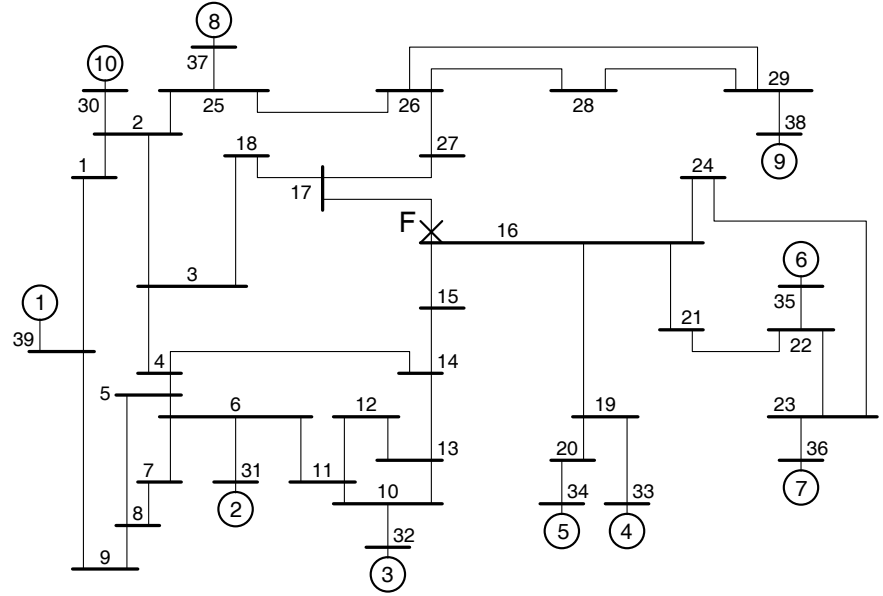

Fig. 1. The New England 39-bus test system (NE system) [19]

$r_{j} \mathrm{e}^{-\mathrm{i} 2 \pi \nu_{j}}$, the corresponding modal dynamics, denoted by $\boldsymbol{g}^{j}\left(\boldsymbol{x}_{k}\right)$, are given by

$$
\begin{aligned}
\boldsymbol{g}^{j}\left(\boldsymbol{x}_{k}\right) & =\lambda_{j}^{k} \varphi_{j}\left(\boldsymbol{x}_{0}\right) \boldsymbol{v}_{j}+\left(\lambda_{j}^{\mathrm{c}}\right)^{k}\left\{\varphi_{j}\left(\boldsymbol{x}_{0}\right) \boldsymbol{v}_{j}\right\}^{\mathrm{c}} \\
& =2 r_{j}^{k}\left[\begin{array}{c}
A_{j 1} \cos \left(2 \pi k \nu_{j}+\alpha_{j 1}\right) \\
\vdots \\
A_{j p} \cos \left(2 \pi k \nu_{j}+\alpha_{j p}\right)
\end{array}\right],
\end{aligned}
$$

where

$$
\begin{aligned}
A_{j i} & =\sqrt{\left(\operatorname{Re}\left[\varphi_{j}\left(\boldsymbol{x}_{0}\right) \boldsymbol{v}_{j}\right]_{i}\right)^{2}+\left(\operatorname{Im}\left[\varphi_{j}\left(\boldsymbol{x}_{0}\right) \boldsymbol{v}_{j}\right]_{i}\right)^{2}}, \\
\tan \alpha_{j i} & =\frac{\operatorname{Im}\left[\varphi_{j}\left(\boldsymbol{x}_{0}\right) \boldsymbol{v}_{j}\right]_{i}}{\operatorname{Re}\left[\varphi_{j}\left(\boldsymbol{x}_{0}\right) \boldsymbol{v}_{j}\right]_{i}} .
\end{aligned}
$$

The notation $\operatorname{Re}\left[\boldsymbol{\varphi}_{j}\left(\boldsymbol{x}_{0}\right) \boldsymbol{v}_{j}\right]_{i}$ stands for the $i$-th component of vector $\operatorname{Re}\left[\varphi_{j}\left(\boldsymbol{x}_{0}\right) \boldsymbol{v}_{j}\right]$. The real part of $\varphi_{j}\left(\boldsymbol{x}_{0}\right) \boldsymbol{v}_{j}$ determines the initial amplitude of modal dynamics, and the imaginary part affects their initial phase. Thus, we can say that a set of oscillatory components $\mathbb{I} \subseteq\{1, \ldots, p\}$ is coherent with respect to Mode $j$ if the amplitude coefficients $A_{j i}$ are the same for all $i \in \mathbb{I}$, and the initial phases $\alpha_{j i}$ are also the same ${ }^{1}$. Then, for coherent identification for Mode $j$, it is sufficient to check both the amplitude coefficients $A_{j i}$ and initial phases $\alpha_{j i}$. Numerically, it is enough to group oscillatory components with similar amplitude coefficient $A_{j i}$ and initial phase $\alpha_{j i}$ as a set of coherent components. When the observables $\left\{\boldsymbol{g}\left(\boldsymbol{x}_{k}\right)\right\}$ contain swing dynamics of generators in a power system, we can find a coherent group of the generators in which they swing together in frequency and phase.

\section{Application to the New England Test System}

We apply the above-described computation of Koopman mode to analyze short-term swing dynamics in the New England 39-bus test system (NE system). The NE system is shown in Fig. 1 and contains the 10 generation units (equivalent 10

\footnotetext{
${ }^{1}$ The definition is strict compablack with the definitions of slow-coherency [2], [6] and near-coherency [7], because it does not admit any finite, constant phase difference of swings. It is easily relaxed and can match the definitions of coherency proposed previously.
} 
synchronous generators, circled numbers in the figure), the 39 buses, and AC transmission lines. Most of the buses have constant active and reactive power loads. The details of the NE system, such as unit rating, line data, and loading conditions, are given in [19].

\section{A. The Swing Equations}

First, we introduce the equations of motion of generators in the NE system. Assume that bus 39 is the infinite bus. The short-term swing dynamics of generators 2-10 are represented by the following nonlinear differential equations, the so-called classical model [22]:

$$
\left.\begin{array}{c}
\frac{\mathrm{d} \delta_{i}}{\mathrm{~d} t}=\omega_{i}, \\
\frac{H_{i}}{\pi f_{\mathrm{b}}} \frac{\mathrm{d} \omega_{i}}{\mathrm{~d} t}=-D_{i} \omega_{i}+P_{\mathrm{m} i}-G_{i i} E_{i}^{2} \\
-\sum_{j=1, j \neq i}^{10} E_{i} E_{j}\left\{G_{i j} \cos \left(\delta_{i}-\delta_{j}\right)+B_{i j} \sin \left(\delta_{i}-\delta_{j}\right)\right\},
\end{array}\right\}
$$

where the integer label $i=2, \ldots, 10$ denotes generator $i$. The variable $\delta_{i}$ is the angular position of rotor in generator $i$ with respect to bus 1 and is in radians [rad]. The variable $\omega_{i}$ is the deviation of rotor speed in generator $i$ relative to that of bus 1 and is in radians per second $[\mathrm{rad} / \mathrm{s}]$. We set the variable $\delta_{1}$ to a constant, because bus 39 is assumed to be the infinite bus. The parameters $f_{\mathrm{b}}, H_{i}, D_{i}, P_{\mathrm{m} i}, E_{i}, G_{i i}, G_{i j}$, and $B_{i j}$ are in per unit system except for $H_{i}$ and $D_{i}$ in seconds [s], and for $f_{\mathrm{b}}$ in Hertz [Hz]. The mechanical input power $P_{\mathrm{m} i}$ to generator $i$ and the internal voltage $E_{i}$ of generator $i$ are normally constant in short-term regime [22]. The parameter $H_{i}$ is the per unit time inertia constant of generator $i$, and $D_{i}$ its damping coefficient. The parameter $G_{i i}$ is the internal conductance, and $G_{i j}+\mathrm{j} B_{i j}$ is the transfer impedance between generators $i$ and $j$. Electrical loads are modeled as passive impedances.

\section{B. Numerical Simulation of the Swing Equations}

The setting of numerical simulation is as follows. The voltage $E_{i}$ and a stable equilibrium $\left(\delta_{i}^{*}, \omega_{i}^{*}=0\right)$ for generator $i$ are fixed using power flow computation [22]. The constants $H_{i}, P_{\mathrm{m} i}$, and power loads are the same as in [19]. The parameter $D_{i}$ is fixed at $0.01 \mathrm{~s}$, and $f_{\mathrm{b}}$ at $60 \mathrm{~Hz}$. The elements $G_{i i}, G_{i j}$, and $B_{i j}$ are calculated using the data in [19] and the power flow computation. All numerical simulations discussed in this paper were performed using MATLAB: the function ode45 is adopted for numerical integration of (7).

We present an example of short-term swing dynamics in the NE system. Fig. 2 shows the time responses of rotor speed deviations $\omega_{i}$ under the initial condition:

$$
\left(\delta_{i}(0), \omega_{i}(0)\right)= \begin{cases}\left(\delta_{i}^{*}+1.5 \mathrm{rad}, 3 \mathrm{rad} / \mathrm{s}\right) & i=8, \\ \left(\delta_{i}^{*}, 0 \mathrm{rad} / \mathrm{s}\right) & \text { else. }\end{cases}
$$

The initial condition physically corresponds to a local disturbance at generator 8 . The generators do not show any steppingout in the figure, that is, they do not show any transient
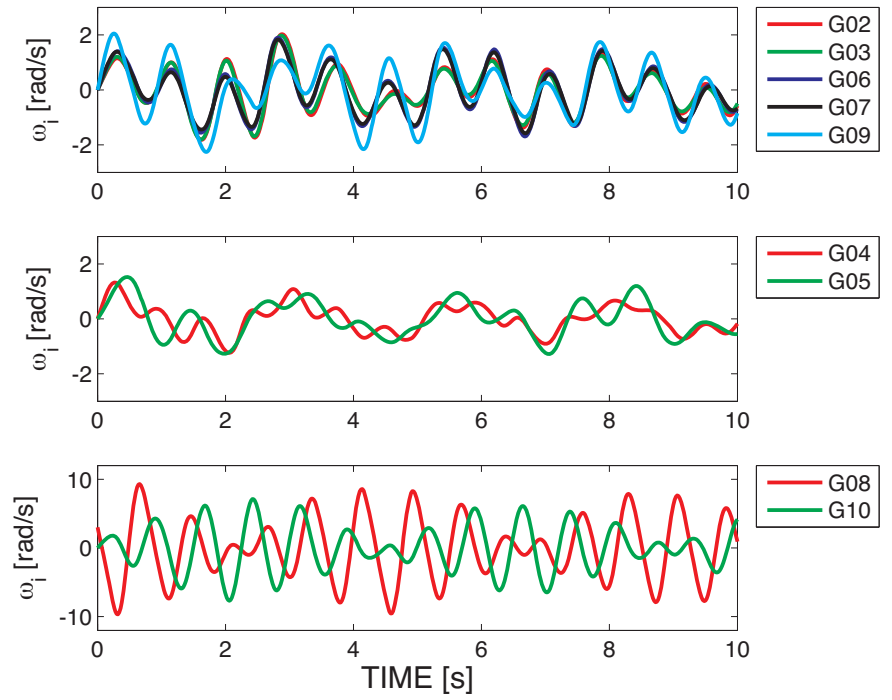

Fig. 2. Coupled swing dynamics of generators $2-10$ in the NE system. These are the trajectories of (7) for the initial condition (8).

instability for the selected disturbance. Generators 2, 3, 6, and 7 show a coherent swing excited by the local disturbance. We call these generators the coherent group. The other generators show incoherent swings in the figure. Generator 9 shows a swing similar in frequency and phase to that of the coherent group, but the swing amplitude is a little larger. Generators 8 and 10 have swings of larger amplitudes than the others, because the initial condition is localized at generator 8 , and the two generators are geographically close. Fig. 3 shows the Discrete Fourier Transform (DFT) of time responses shown in Fig. 2. The computation is done by the function $\mathrm{fft}$ in MATLAB, and the sampling frequency is $50 \mathrm{~Hz}$. The DFT results on generators 8 and 10 have larger magnitudes than the others and have the same shape with two peaks $(1.2 \mathrm{~Hz}$ and $1.4 \mathrm{~Hz}$ ). The coherent group-generators 2, 3, 6, and 7-has the same shape with two peaks $(0.4 \mathrm{~Hz}$ and $1.2 \mathrm{~Hz})$. Since DFT does not consider phase information, we cannot conclude from the DFT result that generators 2, 3, 6, and 7 swing coherently. The shape for generator 9 has three peaks $(0.4 \mathrm{~Hz}, 0.9 \mathrm{~Hz}$, and $1.2 \mathrm{~Hz}$ ) and hence produces a swing similar to that of the coherent group. The other generators, 4 and 5, have shapes different from those mentioned previously.

\section{Decomposition of Coupled Swing Dynamics into Koopman Modes}

We compute the Koopman modes (KMs) for the coupled swing dynamics shown in Fig. 2. To do so, we need to choose the observable $\boldsymbol{g}(\boldsymbol{\delta}, \boldsymbol{\omega})$ where $\boldsymbol{\delta}=\left(\delta_{2}, \ldots, \delta_{10}\right)^{\mathrm{T}}$ and $\boldsymbol{\omega}=\left(\omega_{2}, \ldots, \omega_{10}\right)^{\mathrm{T}}$. The symbol $\mathrm{T}$ indicates complex conjugate transpose in vectors. In this paper we use the variables of rotor speed deviations, $\boldsymbol{\omega}$, as the observable: $\boldsymbol{g}(\boldsymbol{\delta}, \boldsymbol{\omega})=\boldsymbol{\omega}$. This observable has a clear physical meaning in power systems: one measures rotor speeds or frequencies for every generation plant. Using the observable, we compute 

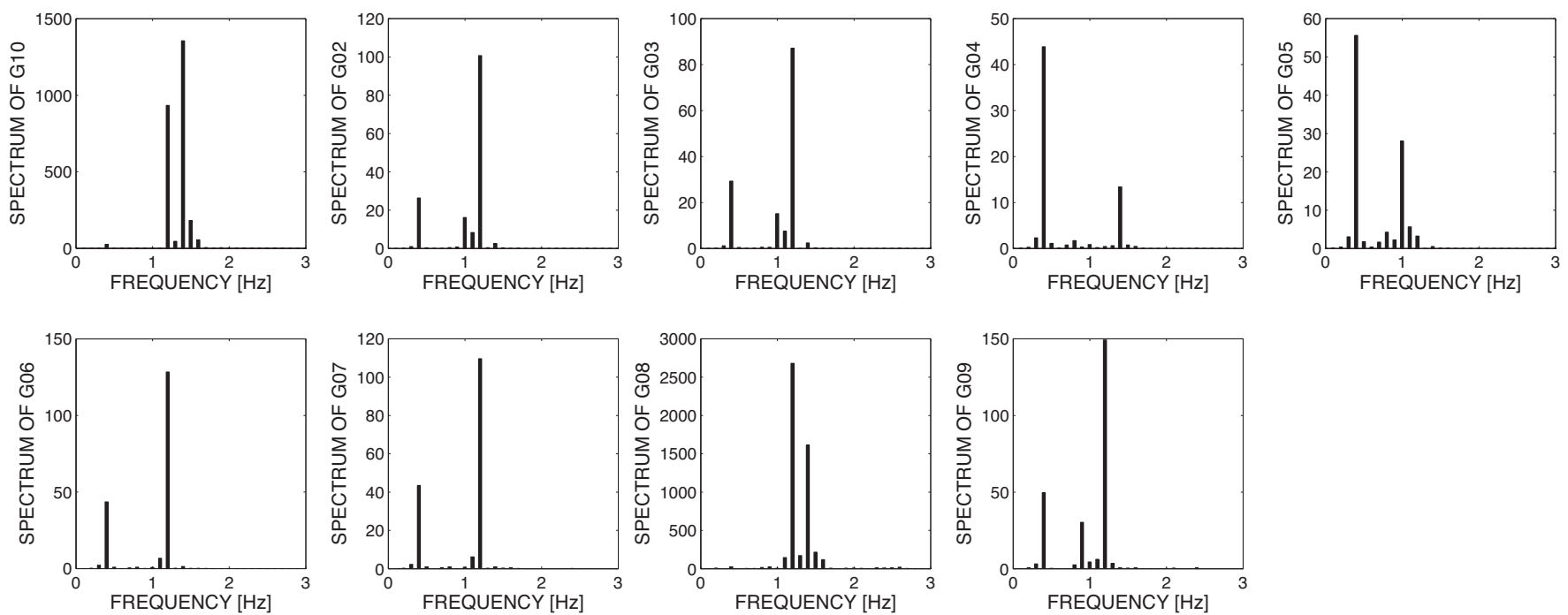

Fig. 3. Numerical discrete Fourier transform of time responses shown in Fig. 2

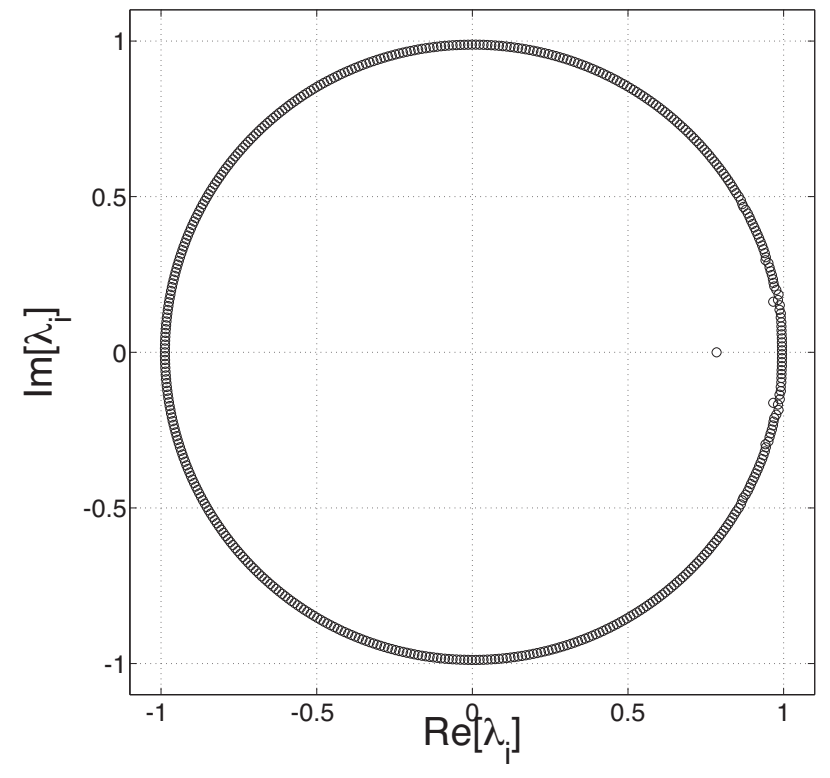

Fig. 4. The empirical Ritz values $\tilde{\lambda}_{j}$

the Koopman eigenvalues and KMs (the empirical Ritz values $\tilde{\lambda}_{j}$ and associated vectors $\tilde{\boldsymbol{v}}_{j}$ ) from the output of numerical simulation. We use the data shown in Fig. 2 that extracts $\{\boldsymbol{\omega}(n T)\}_{n=0}^{N}$ where the sampling period $T=1 /(50 \mathrm{~Hz})$ and the number of samples $N=501$. The implementation of Step (i) in Sec. II-B is explained in Appendix.

Figure 4 shows the empirical Ritz values $\tilde{\lambda}_{j}$. The norm of $r$ in Steps (i) and (iii) is of order $10^{-12}$. Many KMs are obtained and are close to the unit circle $\left|\tilde{\lambda}_{j}\right|=1$. Now let us focus on KMs that have both large growth rates $\left|\tilde{\lambda}_{j}\right|$ and large norms. Such modes represent sustained swing components for the time duration of data and have dominant magnitudes in the
TABLE I

NumericAl RESUltS ON THE KoOPMAN MOdES

\begin{tabular}{ccccc}
\hline $\begin{array}{c}\text { Mode } \\
j\end{array}$ & $\begin{array}{c}\text { Growth Rate } \\
\left|\tilde{\lambda}_{j}\right|\end{array}$ & $\begin{array}{c}\text { Argument }[\mathrm{rad}] \\
\tilde{\theta}_{j}=\operatorname{Im}\left[\ln \tilde{\lambda}_{j}\right]\end{array}$ & $\begin{array}{c}\text { Frequency }[\mathrm{Hz}] \\
\tilde{f}_{j}=\left|\tilde{\theta}_{j}\right| /(2 \pi T)\end{array}$ & $\begin{array}{c}\text { Norm } \\
\left\|\tilde{\boldsymbol{v}}_{j}\right\|\end{array}$ \\
\hline 1 & 1.0011 & \pm 0.1863 & 1.4829 & 1.4299 \\
$\mathbf{2}$ & 0.9994 & \pm 0.1518 & $\mathbf{1 . 2 0 7 7}$ & $\mathbf{3 . 5 2 7 0}$ \\
3 & 0.9990 & \pm 0.1252 & 0.9963 & 0.7767 \\
4 & 0.9974 & \pm 0.0970 & 0.7717 & 0.3687 \\
5 & 0.9972 & \pm 0.1120 & 0.8913 & 0.6734 \\
6 & 0.9969 & \pm 0.2026 & 1.6120 & 1.0893 \\
7 & 0.9965 & \pm 0.0834 & 0.6637 & 0.5271 \\
8 & 0.9963 & \pm 0.1374 & 1.0930 & 1.2944 \\
$\mathbf{9}$ & 0.9963 & \pm 0.1693 & $\mathbf{1 . 3 4 7 4}$ & $\mathbf{4 . 5 0 2 4}$ \\
10 & 0.9958 & \pm 0.0705 & 0.5607 & 0.8343 \\
$\mathbf{1 1}$ & 0.9958 & \pm 0.0447 & $\mathbf{0 . 3 5 5 9}$ & $\mathbf{2 . 5 2 0 9}$ \\
\hline
\end{tabular}

data. Tab. I shows numerical results on Koopman eigenvalues and KMs, which we call Mode 1 to Mode 11. The norm for Mode $j$ is defined as $\left\|\tilde{\boldsymbol{v}}_{j}\right\|=\sqrt{\tilde{\boldsymbol{v}}_{j}^{\mathrm{T}} \tilde{\boldsymbol{v}}_{j}}$. The order of KMs in Tab. I is based on the magnitudes of growth rates. Now we pick up Mode 2, Mode 9, and Mode 11 that have large norms in the table. Fig. 5 shows the KMs $\tilde{\boldsymbol{v}}_{j}$ for Mode $j(j=2,9,11)$. The amplitude coefficients $A_{j i}(j=2,9,11, i=2, \ldots, 10)$ and initial phases $\alpha_{j i}$, defined in (6), are also shown. Fig. 6 shows the modal dynamics (6) for the three KMs. Each KM contains single frequency by construction. For example, Mode 9 with the frequency $1.3474 \mathrm{~Hz}$ has the large amplitude coefficients $A_{j i}$ for generators 8 and 10 (see Fig. 5), and the corresponding modal dynamics shown in Fig. 6(b) are indeed localized at these generators. This KM captures the large swings of generators 8 and 10 observed in Fig. 2, and the mode frequency $1.3474 \mathrm{~Hz}$ almost coincides with one of the dominant frequencies for generators 8 and 10 , that is, $1.4 \mathrm{~Hz}$, shown in Fig. 3. Thus, we can decompose the data on coupled swing dynamics in the NE system into a set of KMs, namely, single-frequency, spatial modes. 

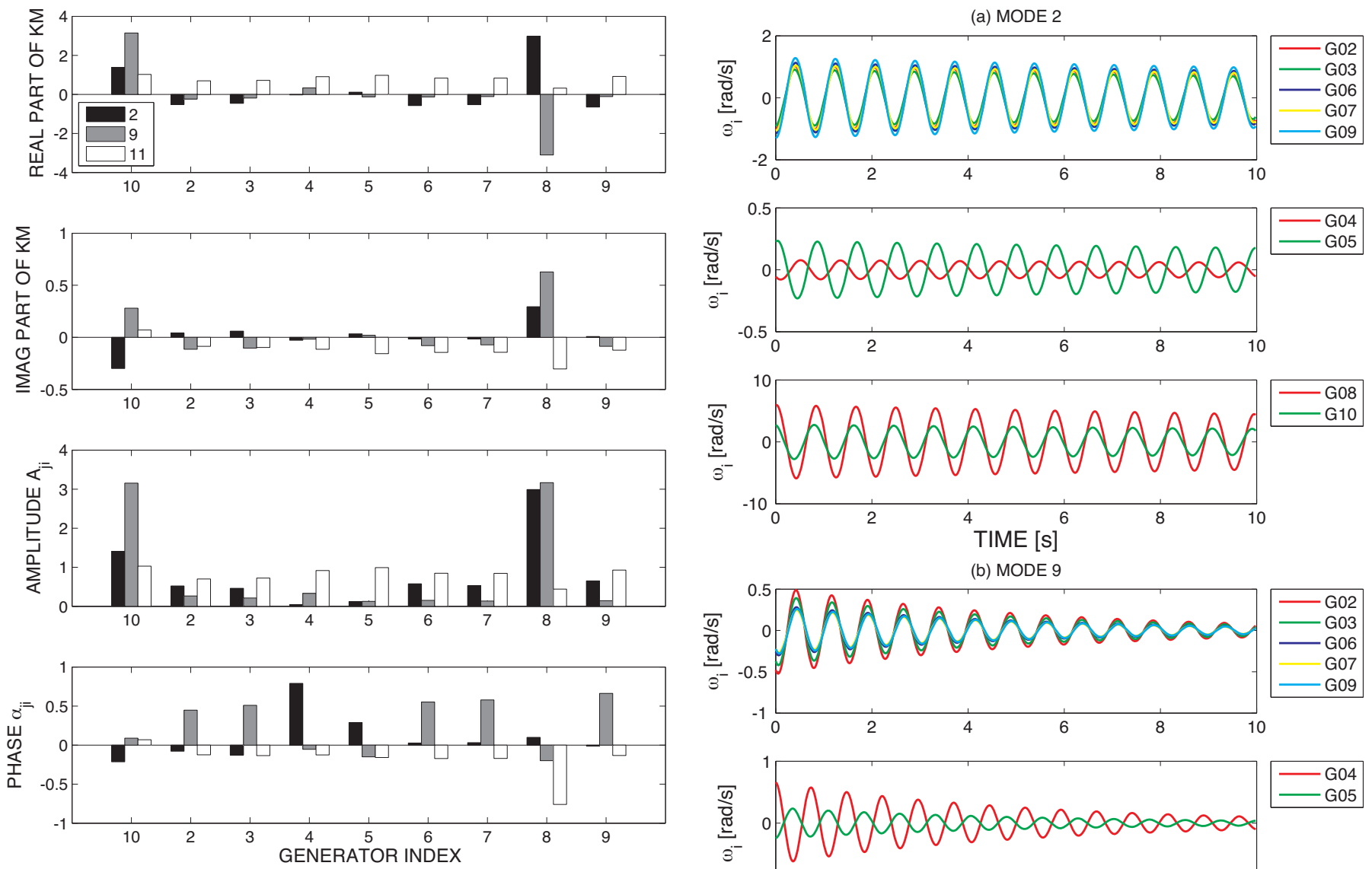

Fig. 5. The Koopman modes $\tilde{\boldsymbol{v}}_{j}(j=2,9,11)$ in Tab. I. The amplitude coefficients $A_{j i}(i=2, \ldots, 10)$ and initial phases $\alpha_{j i}$, defined in (6), are shown.

\section{Coherency Identification Using the Koopman Modes}

The decomposition into KMs makes it possible to extract coherent generators in the coupled swing dynamics. Now let us consider the two KMs, Mode 2 and Mode 11. For Mode 2, the values of amplitude coefficients $A_{j i}$ are close for each of generators 2, 3, 6, 7, and 9, and their initial phases $\alpha_{j i}$ are also close. Hence, generators 2, 3, 6, 7, and 9 show in-phase swings with $1.2077 \mathrm{~Hz}$ (see Fig. 6(a)). For Mode 11, the values of $A_{j i}$ and $\alpha_{j i}$ are close for each of the generators except for generator 8 , and hence they show inphase swings with $0.3559 \mathrm{~Hz}$ (see Fig. 6(c)). Thus, based on the definition of coherency in Sec.II-C, we can say that the set of oscillatory components (generator swings) $\{2,3,6,7,9\}$ is coherent with respect to Mode 2 and Mode 11. These KMs capture the coherent group of generators observed in Fig. 2 (these frequencies, $1.2077 \mathrm{~Hz}$ and $0.3559 \mathrm{~Hz}$, are indeed close to those of the coherent group, $1.2 \mathrm{~Hz}$ and $0.4 \mathrm{~Hz}$ ). In this way, we can identify coherency embedded in the coupled swings using their decomposition into KMs.

Here we used the Arnoldi algorithm to compute the Koopman eigenvalues and KMs. But originally, the KM was defined using projection operation based on $\mathcal{P}^{\nu}$, namely, the Fourier analysis. Now we consider the KMs using $\mathcal{P}^{\nu}$. We use the

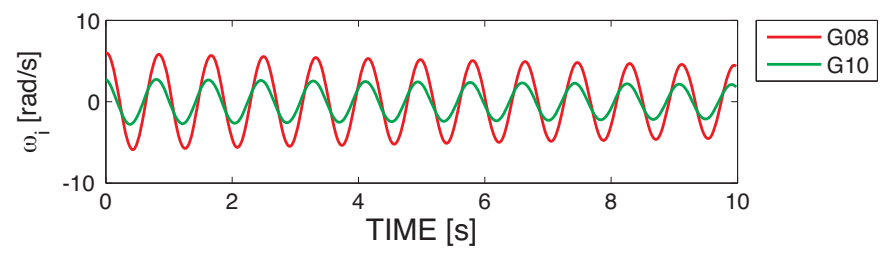
(b) MODE 9
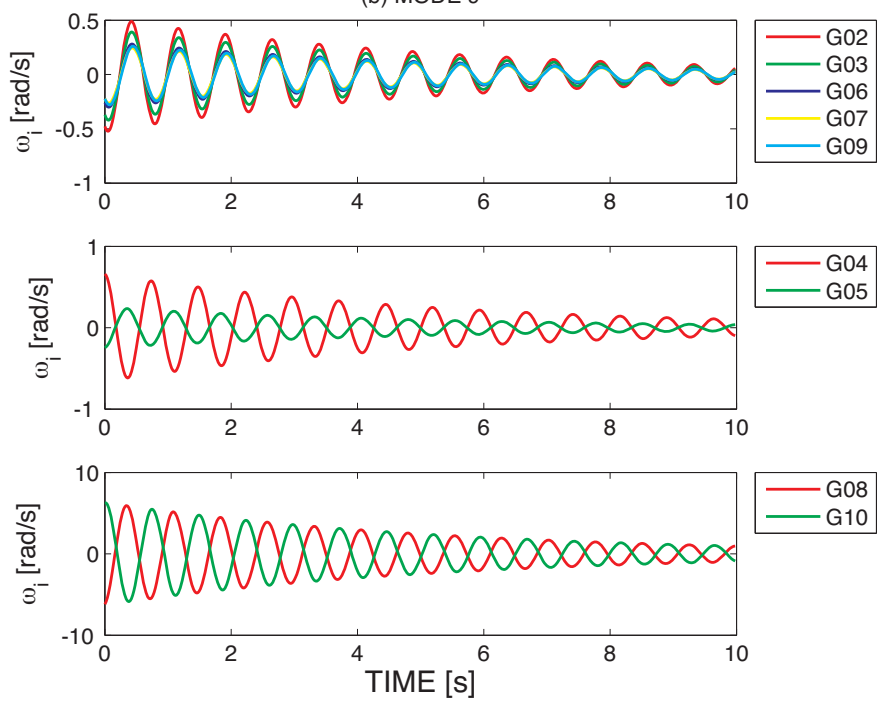

Fig. 6. Mode dynamics (6) of the Koopman modes $\tilde{\boldsymbol{v}}_{1}$ and $\tilde{\boldsymbol{v}}_{2}$

finite-time approximation of (3) from $k=0$ to $N$, which is the number of samples. Fig. 7 shows the numerical results for terms $\varphi_{j}\left(\boldsymbol{x}_{0}\right) \boldsymbol{v}_{j}$ for $\tilde{\nu}_{j}=\tilde{f}_{j} T(j=2,9,11)$. The results for real parts are close to those for KMs in Fig. 5. However, the results for imaginary parts are somewhat different from those in Fig. 5, especially at generator 8. As mentioned in Sec. II-C, the imaginary part of $\varphi_{j}\left(\boldsymbol{x}_{0}\right) \boldsymbol{v}_{j}$ affects the initial phases of associated modal dynamics. Hence we have observed the quantitative differences in the imaginary parts. Also, for the real parts, there are quantitative differences between the results in Figs. 5 and 7. The difference might be due to the assumption of (3) that dynamics are on an attractor. The current analysis is indeed performed for transient dynamics far from an attractor (in this case, a stable equilibrium). However, identification of coherent generations is possible using the Fourier analysis as well. In fact, the coherent swings of generators 2, 3, 6, 7, and 9 are captublack well. 
(c) MODE 11
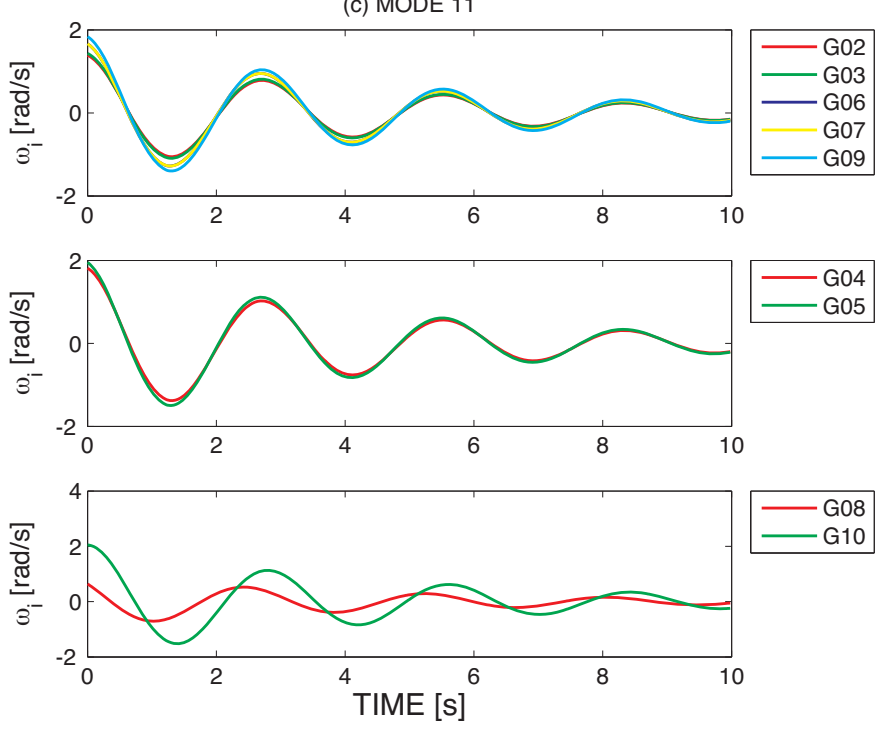

Fig. 6. ( continued)
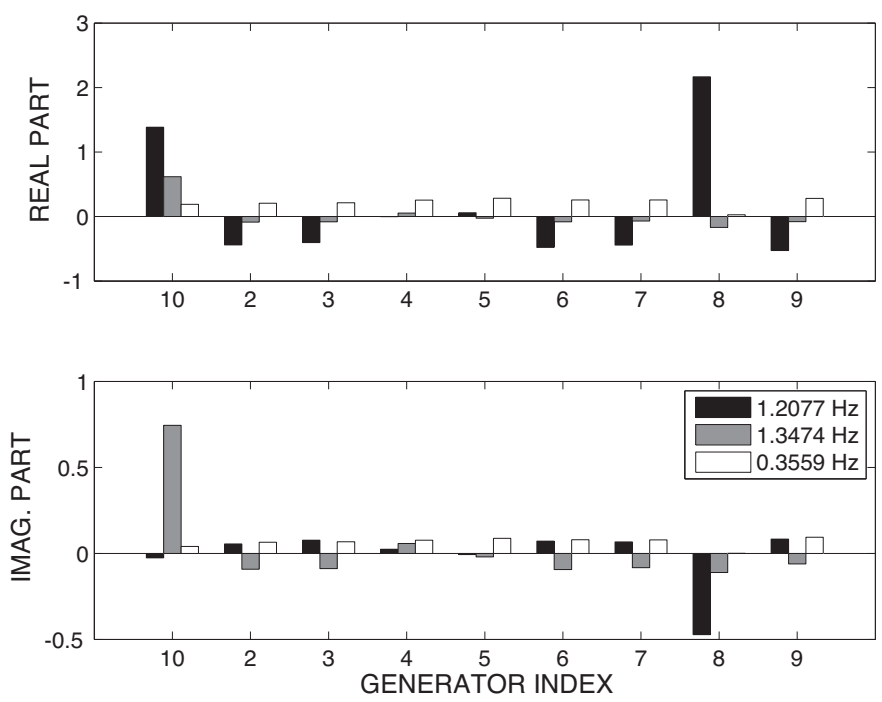

Fig. 7. Numerical results of terms $\varphi_{i}\left(\boldsymbol{x}_{0}\right) \boldsymbol{v}_{i}$ using the projection operator $\mathcal{P}^{\nu}$

\section{SUMMARY}

In this paper, we reported modal analysis of coupled swing dynamics in the New England 39-bus test system (NE system). The computation of Koopman Mode (KM) can be performed purely on finite-time data of dynamics. We show that the modal analysis makes it possible to extract single-frequency (spatial) modes embedded in coupled swing dynamics of the NE system and to identify a coherent group of generators in which they swing together with the in-phase motion. An important advantage of the proposed identification is that we can accurately identify coherency embedded in coupled swings without checking their waveforms. Hence the modal analysis based on the KM provides a systematic and rigorous way for coherency identification and is effective, in particular, when we handle data of coupled swings from a large number of generators, or coherency is invisible in their waveforms. This is the first report of the application of KM to power system analysis. Comparisons with linear global modes and proper orthonormal modes will be presented in a forthcoming archival journal paper [21].

\section{ACKNOWLEDGMENT}

This research is supported in part by the JSPS Postdoctoral Fellowships for Research Abroad.

\section{REFERENCES}

[1] E. H. Abed and P. P. Varaiya, "Nonlinear oscillations in power systems," Electrical Power \& Energy Systems, vol. 6, no. 1, pp. 37-43, January 1984.

[2] J. R. Winkelman, J. H. Chow, B. C. Bowler, B. Avramović, and P. V. Kokotović, "An analysis of interarea dynamics of multi-machine systems," IEEE Transactions on Power Appratuses and Systems, vol. PAS-100, no. 2, pp. 754-763, February 1981.

[3] H. D. Chiang, C. W. Liu, P. P. Varaiya, F. F. Wu, and M. G. Lauby, "Chaos in a simple power system," IEEE Transactions on Power Systems, vol. 8, no. 4, pp. 1407-1417, November 1993.

[4] V. M. Venkatasubramanian and Y. Li, "Analysis of 1996 Western American electric blackouts," in Proceedings of the Bulk Power System Dynamics and Control-VI, Cortina d'Ampezzo, Italy, August 2004, pp. 685-721.

[5] Y. Susuki, I. Mezić, and T. Hikihara, "Global swing instability of multimachine power systems," in Proceedings of the 47th IEEE Conference on Decision and Control, Cancun, Mexico, December 9-11 2008, pp. 2487-2492.

[6] B. Avramović, P. V. Kokotović, J. R. Winkelman, and J. H. Chow, "Area decomposition for electromechanical models of power systems," Automatica, vol. 16, pp. 637-648, November 1980.

[7] S. Sastry and P. Varaiya, "Coherency for interconnected power systems," IEEE Transactions on Automatic Control, vol. AC-26, no. 1, pp. 218226, February 1981.

[8] G. Troullinos and J. Dorsey, "Coherency and model reduction: State space point of view," IEEE Transactions on Power Systems, vol. 4, no. 3, pp. 988-995, August 1989.

[9] Y. Ohsawa and M. Hayashi, "Construction of power system transient stability equivalents using the Lyapunov function," International Journal of Electronics, vol. 50, no. 4, pp. 273-288, April 1981.

[10] M. H. Haque and A. H. M. A. Rahim, "Identification of coherent generators using energy function," IEE Proceedings, Part C, vol. 137, no. 4, pp. 255-260, July 1990.

[11] K. K. Anaparthi, B. Chaudhuri, N. F. Thornhill, and B. C. Pal, "Coherency identification in power systems through principle component analysis," IEEE Transactions on Power Systems, vol. 20, no. 3, pp. 1658-1660, August 2005.

[12] B. O. Koopman, "Hamiltonian systems and transformations in Hilbert space," Proceedings of the National Academy of Sciences of the USA, vol. 17, no. 5, pp. 315-318, May 1931.

[13] K. Peterson, Ergodic Theory. Cambridge: Cambridge University Press, 1983.

[14] A. Lasota and M. C. Mackey, Chaos, Fractals, and Noise: Stochastic Aspects of Dynamics. New York: Springer-Verlag, 1994.

[15] I. Mezić and A. Banaszuk, "Comparison of systems with complex behavior," Physica D, vol. 197, pp. 101-133, 2004.

[16] N. Wiener and A. Wintner, "Harmonic analysis and ergodic theory," American Journal of Mathematics, vol. 63, no. 2, pp. 415-426, April 1941.

[17] I. Mezić, "Spectral properties of dynamical systems, model reduction and decompositions," Nonlinear Dynamics, vol. 41, pp. 309-325, August 2005.

[18] C. W. Rowley, I. Mezić, S. Bagheri, P. Schlatter, and D. S. Henningson, "Spectral analysis of nonlinear flows," Journal of Fluid Mechanics, vol. 641, pp. 115-127, 2009.

[19] M. A. Pai, Energy Function Analysis for Power System Stability. Kluwer Academic Pub., 1989. 
[20] A. G. Phadke, "Synchoronized phasor measurement in power systems," IEEE Computer Applications in Power, vol. 6, no. 2, pp. 10-15, April 1993.

[21] Y. Susuki and I. Mezić, "Nonlinear Koopman modes and coherency identification of coupled swing dynamics," 2009, (submitted).

[22] P. Kundur, Power System Stability and Control. McGraw-Hill, 1994. 